\title{
Multiphoton fluorescence lifetime imaging of chemotherapy distribution in solid tumors
}

\author{
Marjorie Carlson \\ Adrienne L. Watson \\ Leah Anderson \\ David A. Largaespada \\ Paolo P. Provenzano
}




\title{
Multiphoton fluorescence lifetime imaging of chemotherapy distribution in solid tumors
}

\author{
Marjorie Carlson, ${ }^{\mathrm{a}, \mathrm{b}, \dagger}$ Adrienne L. Watson, ${ }^{\mathrm{c}, \dagger}$ Leah Anderson, ${ }^{\mathrm{c}}$ David A. Largaespada, ${ }^{\mathrm{c}}$ and \\ Paolo P. Provenzano a,b,c,d,e,* \\ aUniversity of Minnesota, Department of Biomedical Engineering, Minneapolis, Minnesota, United States \\ bUniversity of Minnesota, Physical Sciences in Oncology Center, Minneapolis, Minnesota, United States \\ 'University of Minnesota, Masonic Cancer Center, Minneapolis, Minnesota, United States \\ dUniversity of Minnesota, Stem Cell Institute, Minneapolis, Minnesota, United States \\ eUniversity of Minnesota, Institute for Engineering in Medicine, Minneapolis, Minnesota, United States
}

\begin{abstract}
Doxorubicin is a commonly used chemotherapeutic employed to treat multiple human cancers, including numerous sarcomas and carcinomas. Furthermore, doxorubicin possesses strong fluorescent properties that make it an ideal reagent for modeling drug delivery by examining its distribution in cells and tissues. However, while doxorubicin fluorescence and lifetime have been imaged in live tissue, its behavior in archival samples that frequently result from drug and treatment studies in human and animal patients, and murine models of human cancer, has to date been largely unexplored. Here, we demonstrate imaging of doxorubicin intensity and lifetimes in archival formalin-fixed paraffin-embedded sections from mouse models of human cancer with multiphoton excitation and multiphoton fluorescence lifetime imaging microscopy (FLIM). Multiphoton excitation imaging reveals robust doxorubicin emission in tissue sections and captures spatial heterogeneity in cells and tissues. However, quantifying the amount of doxorubicin signal in distinct cell compartments, particularly the nucleus, often remains challenging due to strong signals in multiple compartments. The addition of FLIM analysis to display the spatial distribution of excited state lifetimes clearly distinguishes between signals in distinct compartments such as the cell nuclei versus cytoplasm and allows for quantification of doxorubicin signal in each compartment. Furthermore, we observed a shift in lifetime values in the nuclei of transformed cells versus nontransformed cells, suggesting a possible diagnostic role for doxorubicin lifetime imaging to distinguish normal versus transformed cells. Thus, data here demonstrate that multiphoton FLIM is a highly sensitive platform for imaging doxorubicin distribution in normal and diseased archival tissues. @ 2017 Society of Photo-Optical Instrumentation Engineers (SPIE) [DOI: 10.1117/1.JBO.22.11.116010]
\end{abstract}

Keywords: multiphoton microscopy; fluorescence lifetime imaging microscopy; cancer.

Paper 170556R received Aug. 21, 2017; accepted for publication Nov. 8, 2017; published online Nov. $29,2017$.

\section{Introduction}

Despite increasingly promising molecular targeted and immune therapies, small molecule chemotherapeutics remain the primary treatment option for most cancers. In this context, doxorubicin (Adriamycin) is commonly employed to combat many liquid and solid tumors, including carcinomas of the breast, and sarcomas such as malignant peripheral nerve sheath tumors (PNSTs). ${ }^{1-5}$ Although our understanding of its mechanisms of action remains incomplete, doxorubicin is an anthracycline antibiotic that is thought to kill cancer cells through a number of mechanisms that include intercalating between DNA base pairs, inhibiting the progression of topoisomerase II, oxidative stress, mitochondrial dysfunction, DNA torsion, and disruption of chromatin dynamics. ${ }^{6-8}$ Notably, doxorubicin also possesses strong fluorescent properties, ${ }^{9-11}$ which can be utilized to monitor its delivery and distribution in normal tissues and tumors, including human patient samples. ${ }^{12}$

Due to its intrinsic fluorescent properties and small molecular size ( $\sim 550 \mathrm{Da})$, representative of many chemotherapies,

\footnotetext{
*Address all correspondence to: Paolo P. Provenzano, E-mail: pprovenz@umn .edu

†These authors contributed equally
}

doxorubicin has emerged as an ideal reagent for modeling drug delivery in vivo. Early work took advantage of doxorubicin's properties to study its distribution in healthy golden Syrian hamsters in vivo, demonstrating distribution into healthy organs (e.g., heart, lung, liver, and kidney) and nuclear uptake as early as 0.5 min following intravenous administration. ${ }^{13}$ Since then, many studies have utilized doxorubicin, as well as other fluorescent anthracyclines, as a predictor of chemotherapy delivery in cancer using spheroid and grafted tumor models ${ }^{14-18}$ and autochthonous models of disease. ${ }^{19-22}$ Furthermore, many solid tumors present physical barriers to adequate drug delivery resulting in regions of the tumor that are drug-free or experiencing sublethal dosing. ${ }^{23-25}$ This is often noted by a distinct lack of doxorubicin uptake that illustrates significant barriers to molecular transport by diffusion and/or convection. ${ }^{20,23}$ To overcome these barriers to effective drug delivery, recent studies have demonstrated the impact of targeting the tumor stroma to enhance delivery of chemotherapeutics. ${ }^{19-21,26,27}$ As such, methods to image doxorubicin localization in the tumor mass, particularly within cells, and quantify its levels in distinct compartments, are of great value to the studies evaluating approaches to improve drug distribution in tumors.

1083-3668/2017/\$25.00 @ 2017 SPIE 
Fluorescence lifetime imaging microscopy (FLIM) has emerged as a robust method for displaying the spatial distribution of excited state lifetimes. ${ }^{28,29}$ Further, multiphoton FLIM has been utilized to evaluate fundamental aspects of tumor behavior in both live and archival samples. ${ }^{30-36}$ Fluorescence lifetime imaging is particularly useful as it can provide unique identification of a fluorophore and can provide information about molecular structure and a molecule's chemical environment or binding state. ${ }^{37}$ For doxorubicin, lifetime measures have been reported in both the cytoplasm and nucleus of live cells with linear imaging modalities. For instance, examination of HeLa cells demonstrated distinctly shorter doxorubicin lifetimes in the nucleus than in the cytoplasm (both of which are different from doxorubicin in solution), where the shift in lifetime in the nuclei results from binding to DNA. ${ }^{38}$ Although other studies have supported this finding,,${ }^{9,39}$ it has also been reported that doxorubicin lifetime is shorter in the cytoplasm compared with the nucleus. ${ }^{40}$ Furthermore, multiphoton FLIM of live cells has also successfully separated doxorubicin signal from other fluorophores (eGFP and dsRed2), yet to date multiphoton FLIM analysis of doxorubicin in archival formalin-fixed tissues remains incomplete. Here, we describe two-photon processes and lifetime for doxorubicin and fluorescence intensity and lifetime imaging of doxorubicin in archival (formalin-fixed paraffinembedded, FFPE) normal and tumor tissues. We report a significant shift in doxorubicin florescence lifetime in the nucleus versus the cytoplasm and capture localization behavior of doxorubicin that is often elusive in intensity imaging.

\section{Methods}

\subsection{Murine Models of Human Cancer}

This study was approved by the University of Minnesota Institutional Animal Care and Use Committee (IACUC). To generate high-grade PNSTs, we generated genetically engi-

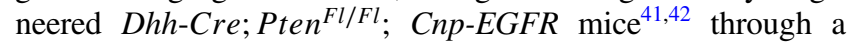
focused breeding scheme. This model is faithful to the fundamental features of human PNSTs ${ }^{41}$ and results in development of plexiform neurofibroma-like lesions and high-grade PNSTs with $100 \%$ penetrance by $\sim 4$ weeks of age. To generate models of pancreatic adenocarcinomas, primary carcinoma cells were grafted into nude $(\mathrm{Nu} / \mathrm{Nu})$ mice. Primary carcinoma cells were established from autochthonous pancreatic tumors from the $\mathrm{Kras}^{\mathrm{LSL}-\mathrm{G} 12 \mathrm{D} /+}$; T p $53^{L S L-R 172 ~ H /+}$; Pdxl-Cre (KPC) genetically engineered murine model, which is a faithful model for human pancreas cancer ${ }^{20,43}$ and harbors the relevant oncogene and tumor suppressor gene mutations associated with human pancreatic cancer, as we have previously described. ${ }^{44}$ Primary carcinoma-associated fibroblasts (CAFs) were isolated by differential trypsinization and magnetic bead depletion of carcinoma cells and immune cell populations. Cells were grown in high glucose Dulbecco-modified eagle's essential medium (Corning) supplemented with 10\% FBS and confirmed to be free of Mycoplasma contamination. To generate tumors, 100,000 carcinoma cells and 100,000 CAFs were mixed into a $2-\mathrm{mg} / \mathrm{mL}$ collagen matrix that was injected subcutaneously in the mouse flank. Tumors developed within 10 days and were harvested 28 days after engraftment. For each model,

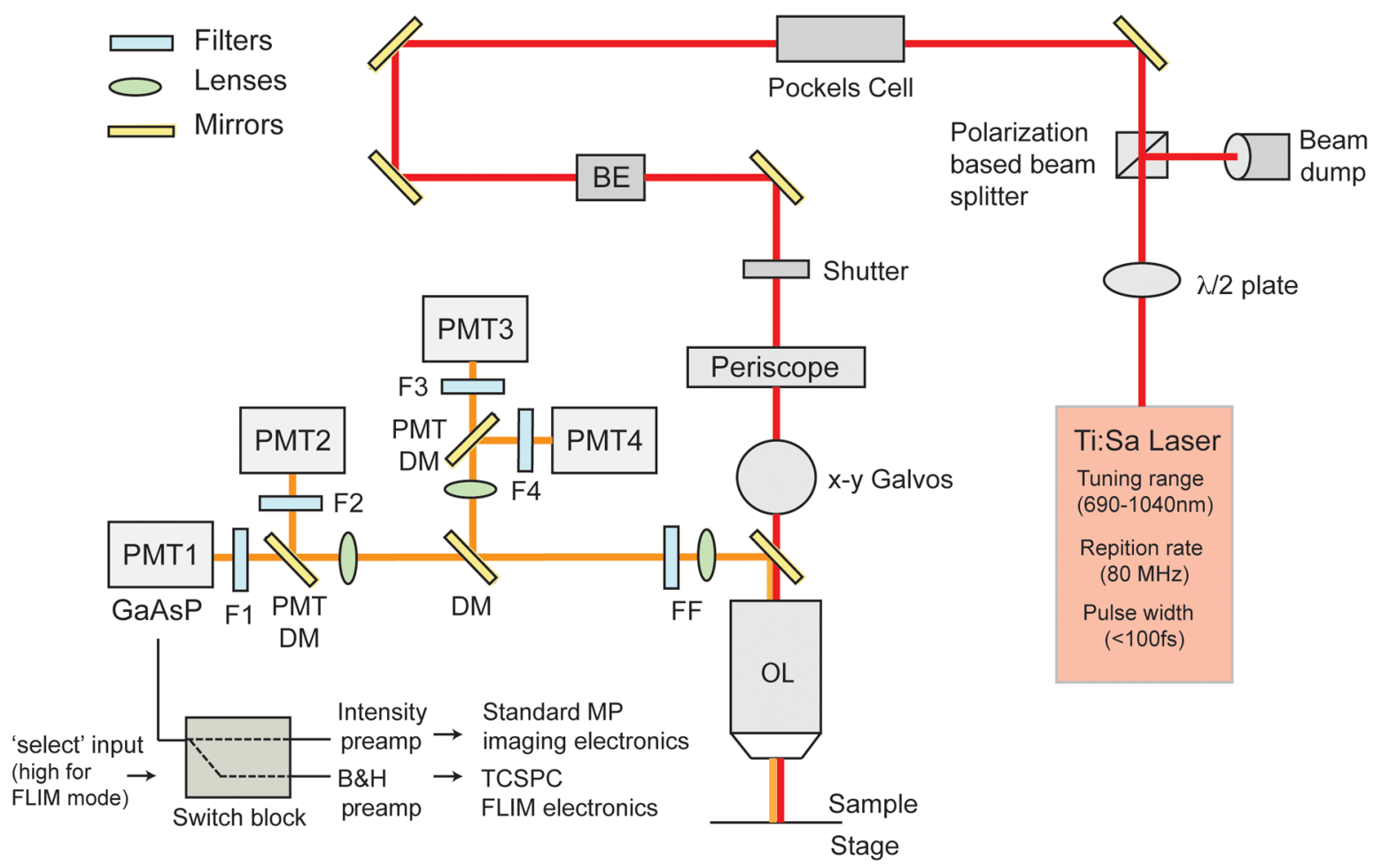

Fig. 1 Multiphoton FLIM setup. Excitation light from the Ti:Sa laser (illustrated in red) is polarized and shaped before entering the galvanometric scanners. Emission signal (illustrated in orange) is captured in the epiconfiguration and sent to a detector array containing multialkali (Hamamatsu) or GaAsP (Hamamatsu model 7422PA-40) PMTs through bandpass filters that include 440/20, 460/50, 525/50, $525 / 70,595 / 50,605 / 70$, and 690/50 filters. Mulitphoton FLIM was performed on this system using the GaAsP PMT connected to an FLIM system employing TCSPC (Becker \& Hickl). 
doxorubicin (20 mg/kg; Sigma) was administered via intraperitoneal (i.p.) injection for PNST models or intravenous (i.v.) injection for pancreatic cancer models $15 \mathrm{~min}$ prior to euthanasia. While under sedation with isoflurane, formalin was injected into the left ventricle 5 min prior to euthanasia. Liver, intestine, and tumor tissues where then harvested and submerged in formalin for at least $24 \mathrm{~h}$ before being processed to generate FFPE histology sections. For doxorubicin imaging, slides were deparaffinized and mounted with Prolong Gold (Life Technologies).

\subsection{Multiphoton Microscopy and Multiphoton Fluorescence Lifetime Imaging}

Doxorubicin solutions or FFPE sections from $n=5$ Dhh-Cre; $P$ ten ${ }^{F l / F l}$; Cnp-EGFR mice or $n=3$ grafted pancreatic tumor models were imaged on a custom-built multiphoton laser scanning microscope (Prairie Technologies/Bruker), where multiphoton imaging relies on a nonlinear dependence on the excitation power and is inherently optically sectioning. A Mai Tai HP Ti:Sapphire laser (Spectra-Physics) that produces 100 -fs pulse widths at an $80-\mathrm{MHz}$ repetition rate to simultaneously generate multiphoton excitation and second harmonic generation (SHG) (Fig. 1) was used. This system was controlled using PrairieView software. Here samples were excited at wavelengths ranging from 730 to $920 \mathrm{~nm}$, and the light focused on the sample using a universal $\mathrm{M}$ plan fluorite infinity corrected nearinfrared $40 \times$ objective $(\mathrm{NA}=0.8)$. Emission was detected in the blue, green, red, and far-red channels (though bandpass filters at either $440 / 20,460 / 50,525 / 50,525 / 70,595 / 50,605 / 70$, or 690/50) with either multialkali (Hamamatsu) or GaAsP (Hamamatsu model 7422PA-40) PMTs. Mulitphoton FLIM was performed on this system using the GaAsP PMT connected to a time-correlated single photon counting (TCSPC) system (Becker \& Hickl). The instrument response function (IRF) was measured using urea crystal to generate the second harmonic signal. Each image of $256 \times 256$ or $512 \times 512$ was acquired using an integration time of 60 to $180 \mathrm{~s}$, with stable photon count rates during image acquisition confirming that photobleaching did not occur. The photon counts from each pixel are used to determine fluorescence lifetime after application of a deconvolution algorithm of the data and a known or estimated instrument response function. ${ }^{37,45}$ Analysis was performed with SPCImage (Becker \& Hickl) with fluorescence decay modeled as a single- or multiexponential by

$I(t)=\sum_{i}^{N} a_{i} \exp ^{\left(t / \tau_{i}\right)}$,

where $a_{i}$ is the fractional contribution, $t$ is the time, and $\tau$ is the fluorescence lifetime. ${ }^{32,37,45,46}$ Here the data were well described by a single exponential function as determined by the minimized $\chi^{2}$ value.

\subsection{Statistical Analysis}

Two group data were analyzed with unpaired $t$-tests. For multigroup comparisons, one-way analysis of variance with a post hoc Tukey-Kramer test was used. For plots of mean lifetime data, boxes are the 25 th to 75 th percentiles and bars represent the maximum and minimum values.

\section{Results and Discussion}

\subsection{Two-Photon Excitation of Doxorubicin}

Doxorubicin is commonly employed to combat many cancers and is frequently utilized to evaluate chemotherapy distribution in tumors and normal tissue due to its fluorescent properties. The one-photon absorption and emission spectra of doxorubicin has been characterized with peak excitation in the $\sim 480$ to $500 \mathrm{~nm}$ range and a broad emission in the range of $\sim 520$ to $720 \mathrm{~nm}$ ( $\lambda_{\text {em }}$ peak in the 580 to $600 \mathrm{~nm}$ range). ${ }^{39,47,48}$ The behavior of doxorubicin in response to multiphoton excitation is less well described. Examining multiphoton excitation across distinct wavelengths ranging from 730 to $920 \mathrm{~nm}$ (Fig. 1), we observed that at equal input power the highest emission intensities result following excitation at 760 to $800 \mathrm{~nm}$ [peak $\lambda_{\text {ex }}=780 \mathrm{~nm}$; Fig. 2(a)]. As expected, emission was detected in the red and far-red channels though bandpass filters at $595 / 50,605 / 70$, and 690/50, and the two-photon nature of doxorubicin excitation was confirmed by examining the nonlinear dependence of fluorescent intensity scaling with the square of the excitation power [Fig. 2(b)]. Thus, doxorubicin has an effective two-photon absorption cross section and, as expected, emits fluorescence into the red/far-red spectrum following two-photon excitation.

\subsection{Fluorescence Lifetime Behavior of Doxorubicin}

The performance of our FLIM setup was initially determined by measuring the IRF and subsequently the fluorescence lifetime of known standards. The IRF was first measured using urea crystals that exhibit strong SHG after interaction with $800-\mathrm{nm}$ pulsed light. SHG is a nonlinear wave mixing process that can occur when light interacts with a material possessing second-order susceptibility. The result is a second-order polarization to generate light at double the excitation frequency (i.e., emission at half of the excitation wavelength) that, in contrast to fluorescent signals, is instantaneous and thus has a zero lifetime. Utilizing the urea crystal-generated IRF, we were able to accurately measure the lifetime YG beads, a well-established standard, to be $2.3 \pm 0.04$ ns (mean \pm s.d.; $n=10$ ), consistent with other reports. ${ }^{49-52}$ Evaluation of the fluorescence lifetime of doxorubicin in PBS solution following multiphoton excitation at $800 \mathrm{~nm}$ revealed a lifetime of $1.01 \pm 0.008 \mathrm{~ns}$ (mean \pm s.d.; $n=7$ ), which is consistent with previous
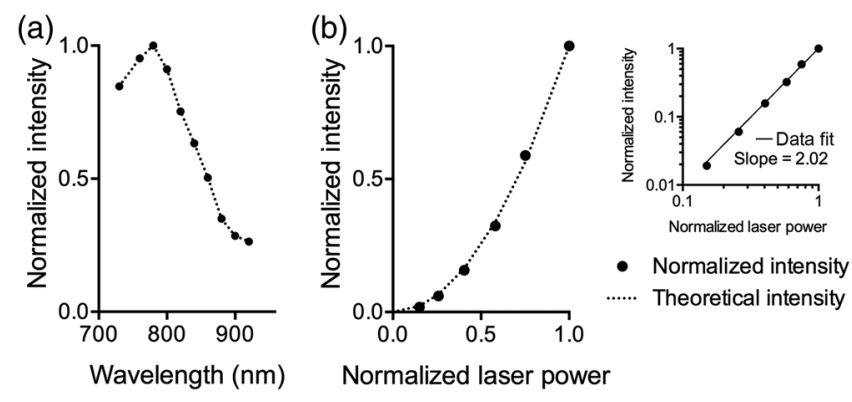

Fig. 2 (a) Emission intensity from doxorubicin excited at wavelengths ranging from 730 to $920 \mathrm{~nm}$. (b) Emission of doxorubicin following excitation at $800 \mathrm{~nm}$ showing the nonlinear dependence of emission on input power that is characteristic of two-photon excitation. Right inset: Log-Log plot of the two-photon-induced fluorescent intensity versus excitation power $\left(R^{2}=0.99\right)$. 
reports using linear excitation modalities,,${ }^{9,11,38,47}$ and was well described with a single exponential model. Thus, multiphoton FLIM provides the capability to robustly generate fluorescence following two-photon excitation and accurately measure its fluorescence lifetime.

\subsection{Doxorubicin Intensity in Archival Nerve Sheath Tumor Sections}

PNSTs are poor-prognosis soft tissue sarcomas that can arise spontaneously or develop in patients with mutations in the NF1 gene that causes neurofibromatosis type $1 .^{53,54}$ Although they are notoriously difficult to treat with both chemical and/ or radiation therapies, these tumors are frequently treated with doxorubicin. ${ }^{1,2,54}$ To generate PNSTs, we employed a genetically engineered murine model of human cancer. PNSTs were generated in Dhh-Cre; Pten $^{F l / F l}$;Cnp-EGFR mice that develop plexiform neurofibroma lesions and highgrade peripheral nerve tumors with $100 \%$ penetrance by $\sim 4$ weeks of age that show strong similarity to their human counterparts. ${ }^{41}$ As such this is an ideal model for studying therapy dynamics. Mice were administered doxorubicin by i.p. injection $15 \mathrm{~min}$ prior to euthanasia. Under anesthesia, mice were rapidly perfused with formalin via intracardiac (i.c.) injection. Tissues were harvested and further fixed in formalin for at least $24 \mathrm{~h}$ and then processed to generate FFPE sections.

Two-photon excitation of doxorubicin in FFPE samples at $\lambda=800 \mathrm{~nm}$ revealed robust doxorubicin fluorescence following fixation and processing. Consistent with analysis of free doxorubicin in solution, maximum emission intensities result following two-photon excitation at 760 to $800 \mathrm{~nm}$ with emission detected in the red and far-red channels though bandpass filters at 595/50, 605/70, and 690/50. In normal FFPE tissues, the doxorubicin signal is strongest from the nucleus, consistent with the intercalation/binding of doxorubicin to DNA, ${ }^{6-8,38}$ with additional signal from the cytoplasm and the extracellular space that vastly exceeds background fluorescence levels observed in regions of poor doxorubicin perfusion or from untreated mice (Fig. 3). Likewise, in PNSTs, examination of the outer boundaries of tumor that are well perfused with doxorubicin revealed that doxorubicin fluorescence is commonly strongest from the nuclei with additional signal from the cytoplasm and the extracellular space [Figs. 3(b), 4(a), and 4(b)]. However, we note that the inner regions of the PNSTs are either not perfused or not well perfused with doxorubicin, consistent with our understanding of poor molecular transport phenomena in solid tumors. ${ }^{20,23,25,27}$ Furthermore, most well perfused regions reflect a spectrum of doxorubicin compartmentalization behavior, including areas in which fluorescent signal from the nuclei is stronger, similar to, and less than that in the cytoplasm [Figs. 3(b1)-3(b2) and 4(c)]. Yet, because the antitumor mechanisms of action of doxorubicin have been described as occurring in both the nucleus and the cytoplasm ${ }^{55-58}$ and extracellular or cytoplasmic doxorubicin may enter the nucleus at later time points, measurement of the total fluorescence intensity in a region is very meaningful, particularly when also considering analysis of molecular transport into a tumor region. ${ }^{20,23}$ However, if detecting doxorubicin in a particular cell compartment or extracellular region is needed, dissecting out the signal localization from fluorescence intensity images can at times be very challenging for instances where the signal intensity is not strongly localized to a distinct cell or extracellular compartment.
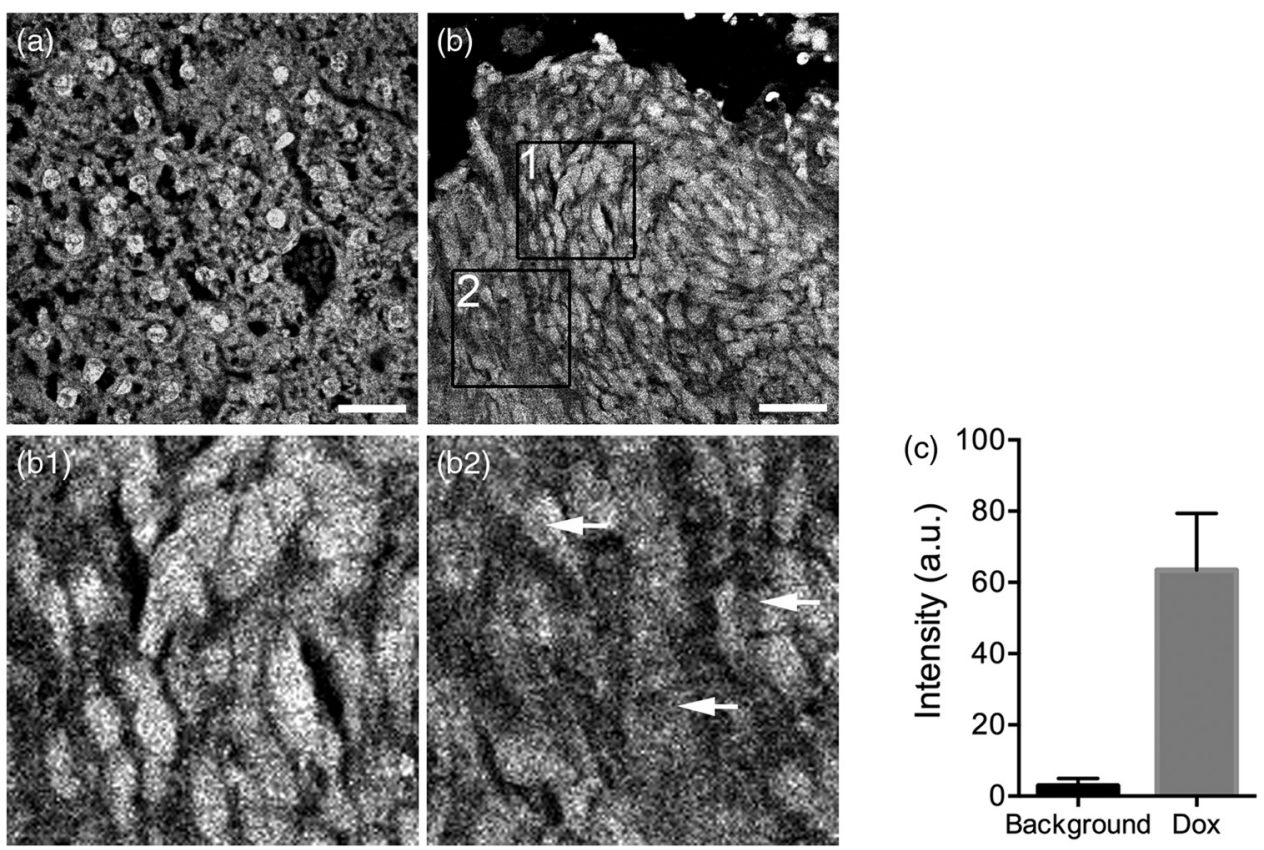

Fig. 3 Multiphoton excitation of doxorubicin at $\lambda_{\mathrm{ex}}=800 \mathrm{~nm}$ in FFPE sections of (a) liver and (b) PNST from Dhh-Cre; Pten ${ }^{F I / F I}$; Cnp-EGFR mice systemically administered $20-\mathrm{mg} / \mathrm{kg}$ doxorubicin $15 \mathrm{~min}$ prior to euthanasia. Boxed regions in 1 and 2 in (b) are magnified in panels b1 and b2, respectively. Arrows in b2 highlight examples of cells with greater doxorubicin signal in the cytoplasm than the nucleus. (c) Quantification of average fluorescence at $\lambda_{\mathrm{ex}}=800 \mathrm{~nm}$ in sections without and with doxorubicin (background and dox, respectively), showing robust doxorubicin fluorescent above background levels (data are mean \pm s.d.). Scale bars $=25 \mu \mathrm{m}$. 


\subsection{Doxorubicin Fluorescence Lifetime Imaging in Solid Tumors}

To test the capability of multiphoton FLIM to capture doxorubicin intensity and lifetime and quantitatively evaluate drug localization, we imaged FFPE archival samples from two distinct tumor models (autochthonous PNSTs and grafted pancreatic adenocarcinomas). Similar to PNST sarcomas, carcinomas of the pancreas have a poor prognosis and respond poorly to chemotherapy. ${ }^{19,20}$ To generate pancreatic tumors, we grafted purified primary carcinoma cells derived from $K P C$ mice into host mice. After 28 days, all tumors reached predefined critical dimensions and doxorubicin was administered systemically 15 min prior to euthanasia, similar to the endpoint protocol used in Dhh-Cre; $\mathrm{Pten}^{\mathrm{Fl} / F l}$; Cnp-EGFR mice. Tissues were again fixed in formalin and processed to generate FFPE sections.
Using multiphoton FLIM with TCSPC, we simultaneously collected both fluorescence intensity and fluorescence lifetime (Figs. 4 and 5). Consistent with standard two-photon excitation imaging of doxorubicin intensity in FFPE tissues from young mice harboring PNSTs (Fig. 3), FLIM data from intestine and liver (tissues that are commonly perfused and impacted by chemotherapy and that are frequently employed to confirm chemotherapy delivery in experimental models), and tumor (PNST) sections imaged at $800 \mathrm{~nm}$ demonstrate that doxorubicin fluorescence is commonly strongest from the nuclei, with additional signal from the cytoplasm and the extracellular space [Figs. 4(a)-4(c)]. Further, heterogeneity in doxorubicin fluorescence within the tumor section remains readily apparent with some cells possessing strong signal from the nuclei while others display more robust intensity from the cytoplasm [Fig. 4(c)]. Extending intensity imaging to the detection of fluorescence
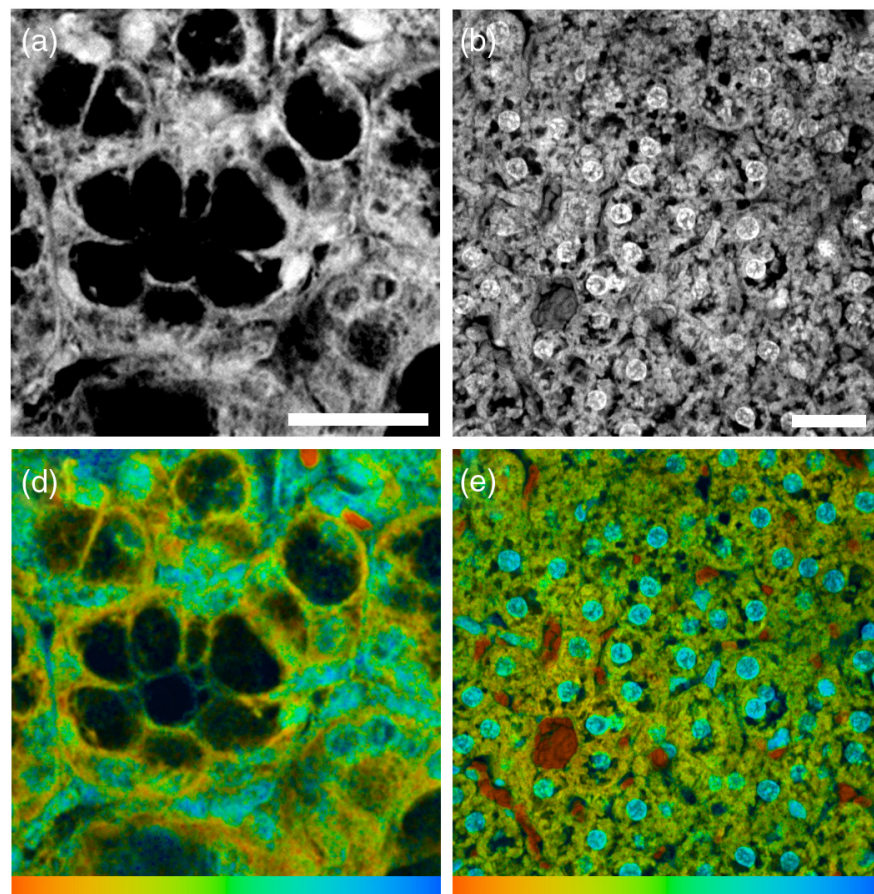

$\tau_{\mathrm{m}}=1.15-1.60(\mathrm{~ns})$

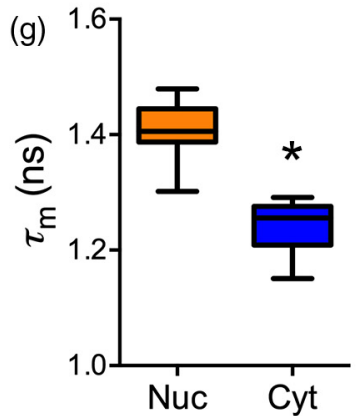

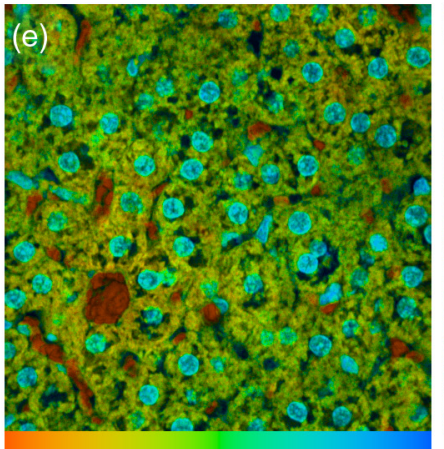

$\tau_{\mathrm{m}}=0.8-1.50(\mathrm{~ns})$

(h)

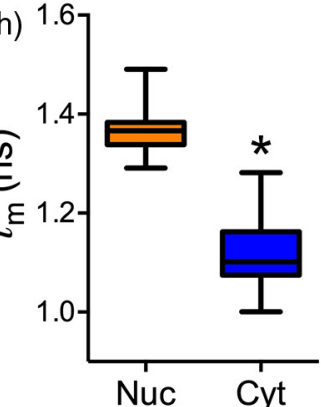

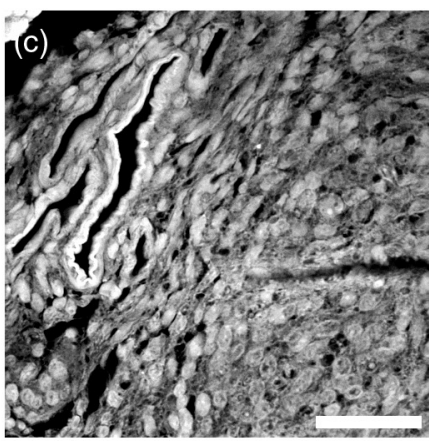

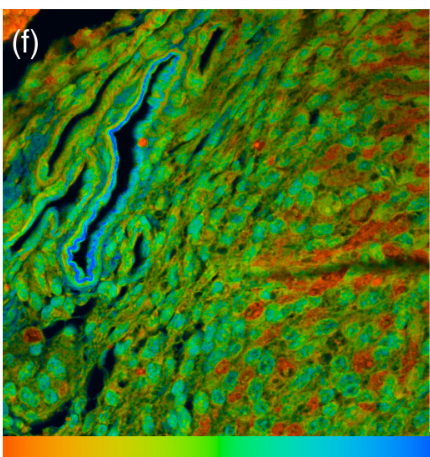

$\tau_{\mathrm{m}}=1.2-1.65(\mathrm{~ns})$

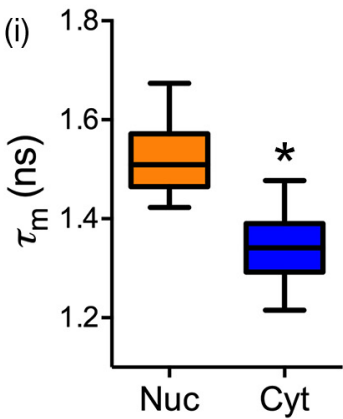

Fig. 4 Multiphoton FLIM imaging of doxorubicin at $\lambda_{\text {ex }}=800 \mathrm{~nm}$ in FFPE sections of $(\mathrm{a}, \mathrm{d}, \mathrm{g})$ intestine, $(\mathrm{b}, \mathrm{e}, \mathrm{h})$ liver, and (c, f, i) PNST from Dhh-Cre; Pten ${ }^{F I / F I}$; Cnp-EGFR mice administered $20-\mathrm{mg} / \mathrm{kg}$ doxorubicin 15 min prior to euthanasia. Using multiphoton FLIM with TCSPC, we simultaneously collected $(a-c)$ intensity and $(d-f)$ fluorescence lifetime data. In all cases, $(g, h, i)$ the lifetime of doxorubicin in nuclei is significantly longer than lifetimes observed in the cytoplasm. For the box and whiskers plots, the box extends from the 25th to 75 th percentiles, while the error bars span the min and max values. ${ }^{*} p<0.05$; scale bars $=25 \mu \mathrm{m}$. 


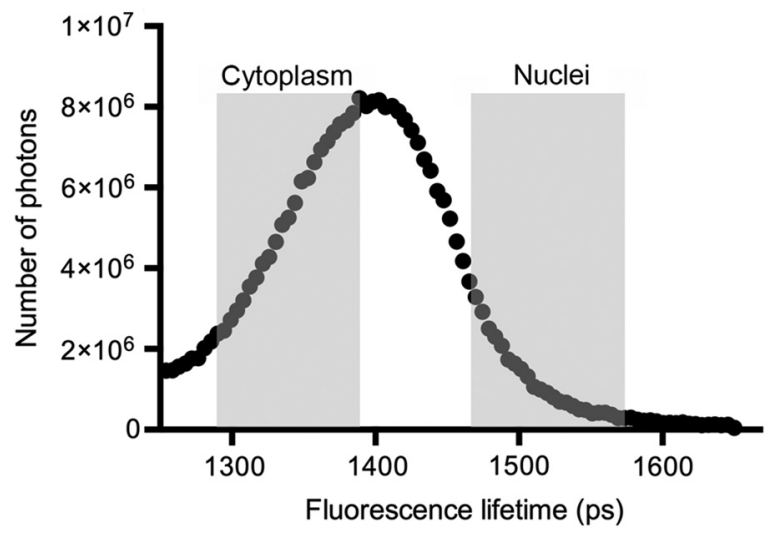

Fig. 5 Quantifying the number of photons for a particular range of lifetimes. Shown here is an example of the photon counts for each fluorescence lifetime in a PNST tumor that is determined from the frequency of a particular lifetime multiplied by the corresponding number of photons in each pixel. Gray boxes represent the region between the 25th and 75th percentiles for the cytoplasmic and nuclei compartments.

lifetimes using multiphoton FLIM reveals strong separation of doxorubicin fluorescence lifetimes in different regions in normal tissue and tumor sections [Figs. 4(d)-4(f)].

The lifetimes for doxorubicin were well described by a single exponential fit, similar to free doxorubicin in solution, but are longer than the lifetime in solution $(\sim 1.2$ to $1.5 \mathrm{~ns}$ versus $\sim 1 \mathrm{~ns}$ ). In particular, a significant separation of doxorubicin lifetimes is observed for doxorubicin in the nucleus versus the cytoplasm in all cases and the distribution of lifetimes in each compartment is quite homogeneous relative to intensity data [consistent with the concentration-independent nature of lifetime imaging; Figs. 4(g)-4(i)]. Interestingly, these characteristics allow for quantification of the relative amount of doxorubicin in each compartment by isolating the number of photons within the range of lifetimes for each compartment (Fig. 5), similar to information obtained by isolating the areas as regions of interest but in a much more efficient way on a larger scale. Furthermore, multicomparison statistical analysis also reveals a significant difference between doxorubicin fluorescence lifetimes in the nuclei of cancer cells versus the nuclei in normal tissues $(p<0.05$ for both tumor versus intestine and tumor versus liver), while lifetimes in the nuclei of the normal tissues are not significantly different ( $p \geq 0.05)$, raising interesting questions about a potential diagnostic role for doxorubicin lifetime imaging to distinguish normal versus transformed cells.

Multiphoton FLIM of FFPE sections from grafted pancreatic ductal adenocarcinomas in adult mice revealed similar behavior to normal tissue and PNSTs in young mice (Figs. 4 and 6). Examination of fluorescence lifetimes from FFPE sections derived from adult intestine, liver, and tumor imaged at $800 \mathrm{~nm}$ shows strong doxorubicin fluorescence in nuclei, with additional signal from the cytoplasm and the extracellular space, with greater heterogeneity in the localization of fluorescence intensity again found in the tumors [Figs. 6(a)-6(c)]. Indeed heterogeneity in doxorubicin fluorescence within the tumor section remains readily apparent, with some cells possessing strong signal from the nuclei while others display more uniform fluorescence or more robust intensity from the cytoplasm [Fig. 6(c)]. Analysis of fluorescence lifetime with multiphoton FLIM reveals strong separation of doxorubicin fluorescence lifetimes in different regions in both normal tissue sections and tumor sections, particularly between nuclei and the cell cytoplasm [Figs. 6(d)-6(i)]. Again, multicomparison statistical analysis reveals a significant difference between doxorubicin fluorescence lifetimes in the nuclei of cancer cells versus the nuclei in normal tissues $(p<0.05$ for both tumor versus intestine and tumor versus liver while liver and intestine are not significantly different, $p \geq 0.05$ ), further supporting the conclusion that there is a distinct behavior for doxorubicin in the nuclei of cancerous cells versus their nontransformed normal counterparts.

The observations here that fluorescence lifetimes in FFPE sections are consistently significantly longer in the nuclei regions than for doxorubicin in the cytoplasm are distinct from findings in some studies using live cancerous cells, ${ }^{9,38,39}$ but they are consistent with other findings in live cancerous cells. ${ }^{40}$ Interestingly, in the cases showing an opposite trend, $, 38,39$ the lifetimes in nuclei are not substantially different between our data in fixed tissues and the published live cells data $(\sim 1.3$ to $1.7 \mathrm{~ns}$ in FFPE sections versus $\sim 1.5$ to $1.8 \mathrm{~ns}$ in live cells). The difference in lifetime in the cytoplasm however is quite different ( $\sim 1.0$ to $1.4 \mathrm{~ns}$ in FFPE sections versus $\sim 1.8$ to $3 \mathrm{~ns}$ in live cells). This may result from a number of factors that include the time that the cells are exposed to doxorubicin, which has been shown to influence lifetimes, ${ }^{38,39}$ and the environmental conditions, such as changes associated with the in vivo versus in vitro condition and fixing and processing the tissues, which are known to impact fluorescence lifetime behavior. ${ }^{37}$ However, here we set out to specifically evaluate doxorubicin in archival sample sections and make comparisons across formalin-fixed archival samples. Notably, the lifetime values here for doxorubicin in the cytoplasm and nuclei are reasonably consistent across each normal tissue from both young and mature mice. The same is true for the values and shifts in distinct tumor types. As such, it is clear that multiphoton FLIM can be effectively implemented on FFPE sections to obtain reliable fluorescence lifetime data for doxorubicin and quantify DNA-bound doxorubicin localized in the cell nucleus.

\section{Conclusion}

Here we demonstrate, for the first time, imaging of doxorubicin intensity and lifetimes with multiphoton excitation and multiphoton FLIM in archival normal tissues and tumors arising in young and mature mice. In each case, FLIM clearly distinguishes between signals arising from cell nuclei versus cytoplasm, which can be used to quantify the relative amount of doxorubicin in each compartment. Furthermore, data from two separate tumor models indicate a shift in lifetime behavior in the nuclei of transformed cells versus their normal counterparts, suggesting a possible role for understanding aberrations in cancerous cells as well as suggesting a possible role for doxorubicin lifetimes to distinguish normal from transformed cells in a diagnostic setting. Moreover, as multiphoton FLIM is demonstrably a highly sensitive platform for imaging doxorubicin distribution, it raises the possibility that FLIM imaging, particularly with nonlinear modalities, may facilitate imaging of additional chemotherapeutics known to possess fluorescent properties such as daunorubicin, paclitaxel, and bleomycin. ${ }^{13,48}$ Thus, multiphoton FLIM is an impactful platform for evaluating chemotherapy signals in normal and diseased tissue and understanding the transport, cellular localization, and kinetics of small molecular chemotherapy. 

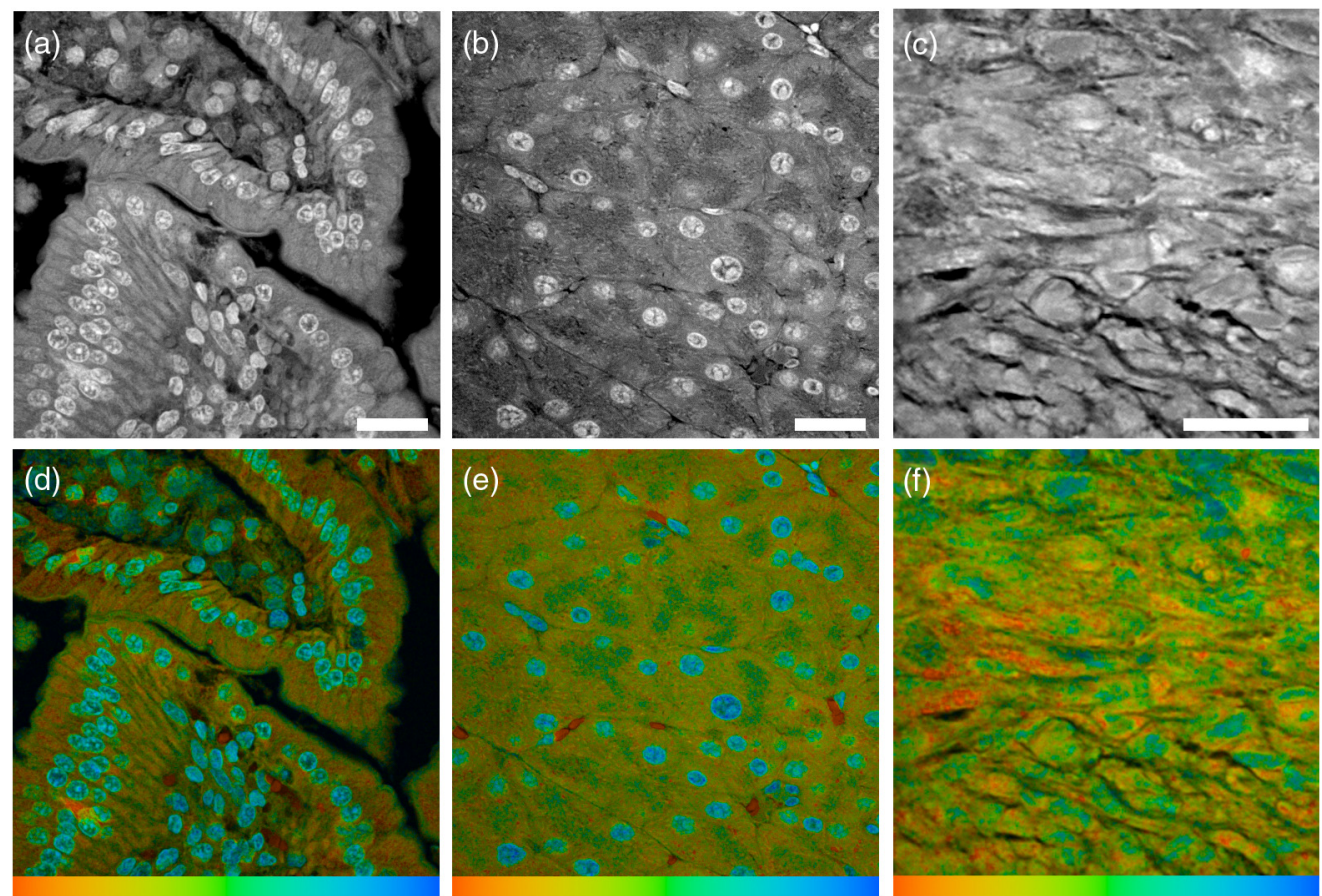

$\tau_{\mathrm{m}}=1.15-1.65(\mathrm{~ns})$

$\tau_{\mathrm{m}}=1.2-1.65(\mathrm{~ns})$

$\tau_{\mathrm{m}}=1.3-1.75(\mathrm{~ns})$
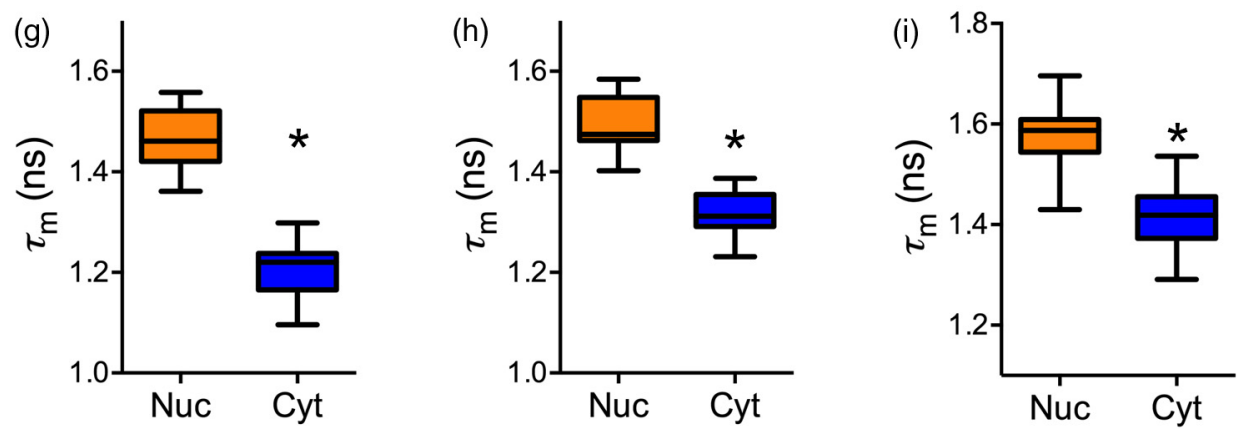

Fig. 6 Multiphoton FLIM imaging of doxorubicin at $\lambda_{\mathrm{ex}}=800 \mathrm{~nm}$ in FFPE sections of $(\mathrm{a}, \mathrm{d}, \mathrm{g})$ intestine, (b, e, h) liver, and (c, f, i) grafted pancreatic ductal adenocarcinoma from mice administered $20-\mathrm{mg} / \mathrm{kg}$ doxorubicin 15 min prior to euthanasia. Using multiphoton FLIM with TCSPC, we simultaneously collected $(a-c)$ intensity and (d-f) fluorescence lifetime data. In all cases, $(g, h, i)$ the lifetime of doxorubicin in nuclei is significantly longer than lifetimes observed in the cytoplasm. For the box and whiskers plots, the box extends from the 25th to 75th percentiles, while the error bars span the min and max values. ${ }^{*} p<0.05$; scale bars $=25 \mu \mathrm{m}$.

\section{Disclosures}

The authors have no relevant financial interests in the article and no other potential conflicts of interest to disclose.

\section{Acknowledgments}

This work was supported by the CDMRP-NFRP (W81XWH15-1-0114 to DL and PPP), the Children's Tumor Foundation (2014A-05-020 to DL and PPP), and grants from the NIH (R01CA181385 to PPP; U54CA210190 University of Minnesota Physical Sciences in Oncology Center Project 2 to PPP; P50CA101955 SPORE career development award to PPP), the American Cancer Society (Research Scholar Grant, RSG-14-171-01-CSM to PPP, Research Professor Award \#123939 to DL), the Randy Shaver Research and Community Fund (PPP), and the UMN Institute for Engineering in Medicine. ALW was supported by the Children's Tumor
Foundation Young Investigator's Award Grant 2011-01-018. We thank Kianna Elahi Gedwillo and Rachel Edwards for assistance with sample preparation and helpful comments. The content of this work is solely the responsibility of the authors and does not necessarily represent the official views of the funding agencies.

\section{References}

1. O. Zehou et al., "Chemotherapy for the treatment of malignant peripheral nerve sheath tumors in neurofibromatosis 1: a 10-year institutional review," Orphanet J. Rare Dis. 8, 127 (2013).

2. J. R. Kroep et al., "First-line chemotherapy for malignant peripheral nerve sheath tumor (MPNST) versus other histological soft tissue sarcoma subtypes and as a prognostic factor for MPNST: an EORTC soft tissue and bone sarcoma group study," Ann. Oncol. 22, 207-214 (2011).

3. J. Lao et al., "Liposomal doxorubicin in the treatment of breast cancer patients: a review," J. Drug Delivery 2013, 1-12 (2013). 
4. Early Breast Cancer Trialists' Collaborative Group, "Effects of chemotherapy and hormonal therapy for early breast cancer on recurrence and 15-year survival: an overview of the randomised trials," Lancet $\mathbf{3 6 5}$, 1687-1717 (2005).

5. M. Xing et al., "Efficacy and cardiotoxicity of liposomal doxorubicinbased chemotherapy in advanced breast cancer: a meta-analysis of ten randomized controlled trials," PloS ONE 10, e0133569 (2015).

6. A. Bodley et al., "DNA topoisomerase II-mediated interaction of doxorubicin and daunorubicin congeners with DNA," Cancer Res. 49, 5969-5978 (1989).

7. F. Yang et al., "Doxorubicin, DNA torsion, and chromatin dynamics," Biochim. Biophys. Acta 1845, 84-89 (2014).

8. D. A. Gewirtz, "A critical evaluation of the mechanisms of action proposed for the antitumor effects of the anthracycline antibiotics adriamycin and daunorubicin," Biochem. Pharmacol. 57, 727-741 (1999).

9. O. Hovorka et al., "Spectral analysis of doxorubicin accumulation and the indirect quantification of its DNA intercalation," Eur. J. Pharm. Biopharm. 76, 514-524 (2010).

10. K. K. Karukstis et al., "Deciphering the fluorescence signature of daunomycin and doxorubicin," Biophys. Chem. 73, 249-263 (1998).

11. S. Shah et al., "Fluorescence properties of doxorubicin in PBS buffer and PVA films," J. Photochem. Photobiol. B 170, 65-69 (2017).

12. J. Lankelma et al., "Doxorubicin gradients in human breast cancer," Clin. Cancer Res. 5, 1703-1707 (1999).

13. M. J. Egorin et al., "Cytofluorescence localization of adriamycin and daunorubicin," Cancer Res. 34, 2243-2245 (1974).

14. R. H. Grantab and I. F. Tannock, "Penetration of anticancer drugs through tumour tissue as a function of cellular packing density and interstitial fluid pressure and its modification by bortezomib," BMC Cancer 12, 214 (2012).

15. A. J. Primeau et al., "The distribution of the anticancer drug doxorubicin in relation to blood vessels in solid tumors," Clin. Cancer Res. 11, 8782-8788 (2005).

16. R. E. Durand, "Slow penetration of anthracyclines into spheroids and tumors: a therapeutic advantage?" Cancer Chemother. Pharmacol. 26, 198-204 (1990).

17. D. J. Kerr et al., "Cytotoxic drug penetration studies in multicellular tumour spheroids," Xenobiotica 18, 641-648 (1988).

18. D. J. Kerr and S. B. Kaye, "Aspects of cytotoxic drug penetration, with particular reference to anthracyclines," Cancer Chemother. Pharmacol. 19, 1-5 (1987).

19. K. P. Olive et al., "Inhibition of hedgehog signaling enhances delivery of chemotherapy in a mouse model of pancreatic cancer," Science 324, 1457-1461 (2009).

20. P. P. Provenzano et al., "Enzymatic targeting of the stroma ablates physical barriers to treatment of pancreatic ductal adenocarcinoma," Cancer Cell 21, 418-429 (2012).

21. M. A. Jacobetz et al., "Hyaluronan impairs vascular function and drug delivery in a mouse model of pancreatic cancer," Gut 62(1), 112-120 (2012).

22. E. S. Nakasone et al., "Imaging tumor-stroma interactions during chemotherapy reveals contributions of the microenvironment to resistance," Cancer Cell 21, 488-503 (2012).

23. P. P. Provenzano and S. R. Hingorani, "Hyaluronan, fluid pressure, and stromal resistance in pancreas cancer," $B r$. J. Cancer 108, 1-8 (2013).

24. R. K. Jain and T. Stylianopoulos, "Delivering nanomedicine to solid tumors," Nat. Rev. Clin. Oncol. 7, 653-664 (2010).

25. V. P. Chauhan et al., "Delivery of molecular and nanoscale medicine to tumors: transport barriers and strategies," Ann. Rev. Chem. Biomol. Eng. 2, 281-298 (2011).

26. B. Diop-Frimpong et al., "Losartan inhibits collagen I synthesis and improves the distribution and efficacy of nanotherapeutics in tumors," Proc. Natl. Acad. Sci. U.S.A. 108, 2909-2914 (2011).

27. V. P. Chauhan et al., "Angiotensin inhibition enhances drug delivery and potentiates chemotherapy by decompressing tumour blood vessels," Nat. Commun. 4, 2516 (2013).

28. J. R. Lakowicz et al., "Fluorescence lifetime imaging," Anal. Biochem. 202, 316-330 (1992).

29. E. B. van Munster and T. W. Gadella, "Fluorescence lifetime imaging microscopy (FLIM)," Adv. Biochem. Eng./Biotechnol. 95, 143-175 (2005).
30. P. P. Provenzano et al., "Collagen density promotes mammary tumor initiation and progression," BMC Med. 6, 11 (2008).

31. P. P. Provenzano et al., "Nonlinear optical imaging and spectral-lifetime computational analysis of endogenous and exogenous fluorophores in breast cancer," J. Biomed. Opt. 13, 031220 (2008).

32. P. P. Provenzano, K. W. Eliceiri, and P. J. Keely, "Shining new light on 3D cell motility and the metastatic process," Trends Cell Biol. 19, 638648 (2009).

33. A. Janssen et al., "Intravital FRET imaging of tumor cell viability and mitosis during chemotherapy," PLOS ONE 8, e64029 (2013).

34. M. Nobis et al., "Intravital FLIM-FRET imaging reveals dasatinibinduced spatial control of SRC in pancreatic cancer," Cancer Res. 73, 4674-4686 (2013).

35 . V. Novitskaya et al., "Integrin $\alpha 3 \beta 1-\mathrm{CD} 151$ complex regulates dimerization of ErbB2 via RhoA," Oncogene 33, 2779-2789 (2014).

36. G. J. Bakker et al., "Fluorescence lifetime microscopy of tumor cell invasion, drug delivery, and cytotoxicity," Methods Enzymol. 504, 109-125 (2012).

37. J. R. Lakowicz, Principles of Fluorescence Spectroscopy, Springer, New York (2006).

38. N. T. Chen et al., "Probing the dynamics of doxorubicin-DNA intercalation during the initial activation of apoptosis by fluorescence lifetime imaging microscopy (FLIM)," PLoS ONE 7, e44947 (2012).

39. P. Weber, M. Wagner, and H. Schneckenburger, "Cholesterol dependent uptake and interaction of doxorubicin in MCF-7 breast cancer cells," Int. J. Mol. Sci. 14, 8358-8366 (2013).

40. X. Dai et al., "Fluorescence intensity and lifetime imaging of free and micellar-encapsulated doxorubicin in living cells," Nanomed. Nanotechnol. Biol. Med. 4, 49-56 (2008).

41. V. W. Keng et al., "Conditional inactivation of Pten with EGFR overexpression in Schwann cells models sporadic MPNST," Sarcoma 2012, $1-12$ (2012).

42. V. W. Keng et al., "PTEN and NF1 inactivation in Schwann cells produces a severe phenotype in the peripheral nervous system that promotes the development and malignant progression of peripheral nerve sheath tumors," Cancer Res. 72, 3405-3413 (2012).

43. S. R. Hingorani et al., "Trp5 $3^{\mathrm{R} 172 \mathrm{H}}$ and $\mathrm{Kras}^{\mathrm{G} 12 \mathrm{D}}$ cooperate to promote chromosomal instability and widely metastatic pancreatic ductal adenocarcinoma in mice," Cancer Cell 7, 469-483 (2005).

44. A. Ray et al., "Anisotropic forces from spatially constrained focal adhesions mediate contact guidance directed cell migration," Nat. Commun. 8, 14923 (2017).

45. Y. S. Sun, R. N. Day, and A. Periasamy, "Investigating protein-protein interactions in living cells using fluorescence lifetime imaging microscopy," Nat. Protoc. 6, 1324-1340 (2011).

46. P. P. Provenzano, K. W. Eliceiri, and P. J. Keely, "Multiphoton microscopy and fluorescence lifetime imaging microscopy (FLIM) to monitor metastasis and the tumor microenvironment," Clin. Exp. Metastasis 26, 357-370 (2009).

47. P. Changenet-Barret et al., "Unravelling molecular mechanisms in the fluorescence spectra of doxorubicin in aqueous solution by femtosecond fluorescence spectroscopy," Phys. Chem. Chem. Phys. 15, 2937-2944 (2013).

48. N. S. H. Motlagh et al., "Fluorescence properties of several chemotherapy drugs: doxorubicin, paclitaxel and bleomycin," Biomed. Opt. Express 7, 2400-2406 (2016).

49. E. B. van Munster et al., "Combination of a spinning disc confocal unit with frequency-domain fluorescence lifetime imaging microscopy," Cytometry A 71, 207-214 (2007).

50. K. Drozdowicz-Tomsia et al., "Multiphoton fluorescence lifetime imaging microscopy reveals free-to-bound NADH ratio changes associated with metabolic inhibition," J. Biomed. Opt. 19, 086016 (2014).

51. M. C. Skala et al., "In vivo multiphoton fluorescence lifetime imaging of protein-bound and free nicotinamide adenine dinucleotide in normal and precancerous epithelia," J. Biomed. Opt. 12, 024014 (2007).

52. D. K. Bird et al., "Metabolic mapping of MCF10A human breast cells via multiphoton fluorescence lifetime imaging of the coenzyme NADH," Cancer Res. 65, 8766-8773 (2005).

53. K. Staser, F. C. Yang, and D. W. Clapp, "Pathogenesis of plexiform neurofibroma: tumor-stromal/hematopoietic interactions in tumor progression," Апnи. Rev. Pathol. 7, 469-495 (2012). 
54. V. C. Williams et al., "Neurofibromatosis type 1 revisited," Pediatrics 123, 124-133 (2009).

55. A. G. Patel and S. H. Kaufmann, "Cancer how does doxorubicin work?" Elife 1, e00387 (2012).

56. S. W. Rabkin and P. Sunga, "The effect of doxorubicin (adriamycin) on cytoplasmic microtubule system in cardiac-cells," J. Mol. Cell Cardiol. 19, 1073-1083 (1987).
57. B. Denard, C. Lee, and J. Ye, "Doxorubicin blocks proliferation of cancer cells through proteolytic activation of CREB3L1," Elife 1, e00090 (2012).

58. C. F. Thorn et al., "Doxorubicin pathways: pharmacodynamics and adverse effects," Pharmacogenet. Genom. 21, 440-446 (2011).

Biographies for the authors are not available. 